\title{
Silicon photonic waveguides for different wavelength regions
}

\author{
G Z Mashanovich ${ }^{1}$, M Milosevic ${ }^{2}$, P Matavulj ${ }^{2}$, S Stankovic ${ }^{2}$, B Timotijevic ${ }^{1}$, P Y Yang ${ }^{1}$, E J Teo ${ }^{3}$, M B H \\ Breese $^{3}$, A A Bettiol ${ }^{3}$ and G T Reed ${ }^{1}$ \\ ${ }^{1}$ Advanced Technology Institute, University of Surrey, Guildford, Surrey, UK \\ ${ }^{2}$ Faculty of Electrical Engineering, University of Belgrade, Serbia \\ ${ }^{3}$ Department of Materials Science and Engineering, National University of Singapore, Singapore
}

\begin{abstract}
In this paper we present our work on three silicon waveguide structures that are suitable for three different wavelength regions: near-, mid- and far-infrared. Design rules for standard rib SOI waveguides are given. Both single mode and polarisation independence in these waveguides are discussed. A hollow core waveguide suitable for gas sensing applications in the mid-infrared wavelength region is also analysed. Finally, fabrication and experimental results for free standing waveguides, which may find application in the mid and perhaps far-infrared wavelength region, are presented.
\end{abstract}

\section{Introduction}

The essential building block of every photonic circuit is the optical waveguide. The first silicon waveguides were reported in the 1980s, in silicon on doped silicon [1, 2], silicon on sapphire [3], silicon germanium [4], and Silicon on Insulator (SOI) [5, 6]. The SOI platform, first reported in 1989, has by far, become the most popular of the four waveguide systems. As SOI is extensively used in the microelectronics industry, it is also one of the most promising materials for optical/electrical integration. The research in waveguiding in silicon started with planar waveguides [7], and continued with large rib waveguides [8]. Recently, there has been a trend to reduce waveguide dimensions and consequently both small rib [9] and strip waveguides [e.g. 10, 11, $12,13]$ have been investigated. In this paper, guidelines for single mode and birefringence free conditions for relatively small rib waveguides are discussed. The influence of the oxide stress on a $1.35 \mu \mathrm{m}$ high rib waveguide is analysed.

Conventional optical waveguides, based on the total internal reflection, have a solid core, made of material with a higher refractive index than that of the surrounding cladding. Although widely used and still preferred in integrated optics, these waveguides suffer from all the shortcomings related to the propagation of light through a solid medium, such as dispersion and temperature dependence of the refractive index. In order to avoid these problems, hollow-core waveguides have been proposed ([14], [15], [16], [17]). The hollow-core waveguides, as the name suggests, have an air-filled core surrounded by a solid cladding comprised of a multilayer coating, designed to confine the propagating optical signal within the core. As the light propagates through the air, there is virtually no dispersion nor problems with temperature dependence of the refractive index. Hollow-core waveguides can be also very useful for sensing applications as their core can be filled with gases or liquids. Other potential applications of these waveguides include tight turning radii and high power transmission.

Research and design of the silicon-based photonic integrated circuits (PICs) has been primarily focused on devices optimised for $1.55 \mu \mathrm{m}$ wavelength, utilised in telecommunications. However, for other applications, such as sensing, imaging or medical applications, wavelengths in the mid-wave- (MWIR) or long-wave infrared (LWIR) spectrum are of interest $[18,19]$. The 3-5 $\mu \mathrm{m}$ wavelength range is particularly interesting because a number of gases have absorption bands in that region. In addition, there is an atmospheric transmission window between 3 and $5 \mu \mathrm{m}$ which enables free space optical communications and thermal imaging applications in both civil and military situations as well as the development of infrared countermeasures for homeland security. As silicon is low loss from 1.2-8 $\mu \mathrm{m}$ and from 24-200 $\mu \mathrm{m}$ [18], a silicon waveguide with air cladding would be also potentially low loss in these two wavelength windows. In this paper we present such a silicon waveguide structure, the free standing waveguide, which has been fabricated by proton beam writing and subsequent electrochemical etching. 
Three silicon waveguide structures with three different geometries, fabricated by three different techniques, and aimed to be used in three different wavelength ranges are presented in this paper, showing the versatility and potential applicability of the silicon waveguide technology.

\section{Rib waveguides}

Unlike silica waveguides which can be relatively easily designed to be single mode, SOI waveguides with dimensions larger than a few hundred nanometres in cross-section will support multiple modes. Such multimode waveguides are usually undesirable in photonic circuits as their operation can be seriously compromised by the presence of multiple modes.

It has been shown, however, that large rib waveguides in SOI could be designed to be monomodal [20]. These waveguides have been studied extensively by a number of researchers [e.g. 21, 22, 23, 24] to find single mode behaviour and low loss propagation. Large rib waveguides are interesting because they are multi-micron in cross sectional dimensions (of the order of $5 \mu \mathrm{m}$ ) facilitating low-loss coupling to and from optical fibres. Soref et al. [20] first proposed a simple expression for the single-mode condition (SMC) of such waveguides:

$$
\left.\frac{W}{H} \leq 0.3+\frac{r}{\sqrt{1-r^{2}}} \quad \text { (for } 0.5<r<1\right)
$$

where $r$ is the ratio of slab height to overall rib height, and $W / H$ is the ratio of waveguide width to overall rib height. Their analysis of the waveguides was limited to shallow etched ribs $(r>0.5)$ and the waveguide dimensions were assumed to be larger than the operating wavelength. The analysis was based on the assumption that high order vertical modes (i.e. modes other than the fundamental mode) confined under the rib, were coupled to the outer slab region during propagation, therefore yielding high propagation losses for the higher order modes. Thus the waveguides behave as single mode waveguides, as all other modes are lost.

Chan et al. [9] produced single mode and polarisation independence equations for relatively small rib waveguides:

$$
\begin{aligned}
& \frac{W}{H} \leq 0.05+\frac{(0.94+0.25 H) r}{\sqrt{1-r^{2}}} \quad \text { for } 0 \leq r \leq 0.5 \text { and } 1.0 \leq H \leq 1.5 \mu m \\
& D_{\min }=0.06 \times 10^{-6}+0.556 H
\end{aligned}
$$

Equation (2) defines the quasi-TM single mode boundary, and hence provides guidance on the geometric limitations to retain single mode behaviour, whilst equation (3) defines the minimum etch depth $D_{\min }$ required to obtain polarisation independence.

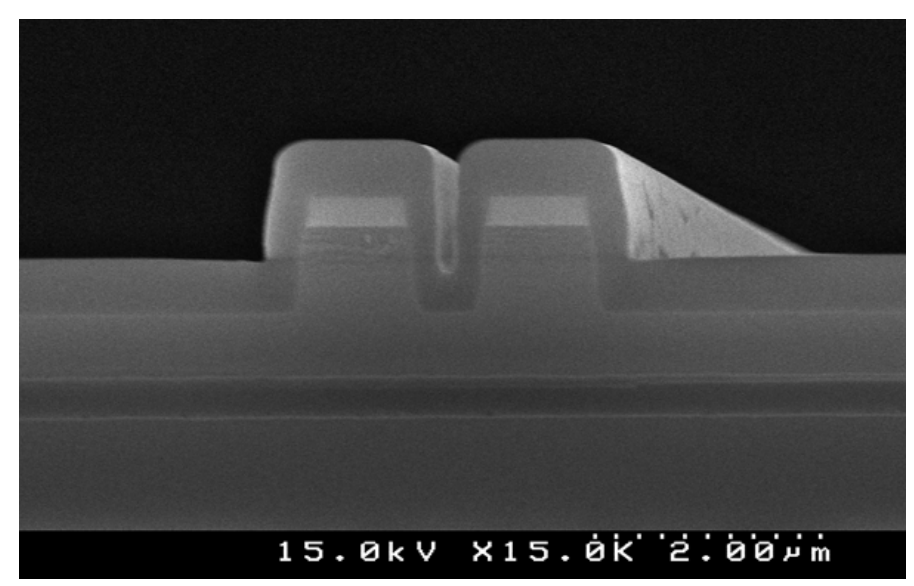

Figure 1. SEM of a directional coupler made of two rib waveguides with top oxide cover 
The guidelines of equations (2) and (3) are for waveguides with an air top cladding. However, an upper cladding layer is often deposited to reduce the influence of surface contamination, to passivate the surface, or to provide electrical isolation. For SOI, this layer is usually $\mathrm{SiO}_{2}$ (figure 1), although nitride and polymer layers are also used in some cases. Therefore, the single mode and birefringence free conditions also need to be determined for waveguides with such claddings, not only because oxide cladding has different refractive index than air but also because it causes stress in the waveguide structure, therefore changing the effective refractive indices for TE and TM polarisations. The structure we analyse in this section is a rib waveguide with the height of $H=1.35 \mu \mathrm{m}$, a variable waveguide width $W$ and etch depth $\mathrm{D}$, which has the following refractive indices: $\mathrm{n}_{\text {core }}=3.4764, \mathrm{n}_{\text {top_cladding }}=\mathrm{n}_{\text {buried_oxide }}=1.444$. The simulations were performed by using a 3D semi-vectorial BPM method and verified by 2D FEM modelling. The method used was to calculate the effective indices for the fundamental and the first two higher order modes, for both polarisations, over a range of waveguide dimensions. It is assumed that the oxide cladding is thick $(>1 \mu \mathrm{m})$. Simulations confirm again that the TM mode is the most critical for multimode behaviour since the single mode (SM) curve for the TM polarisation is below the corresponding curve for the TE polarisation. Fitting the data points to a second order polynomial, the SM condition determined for the TM mode can be expressed as:

$$
W \leq 2.86 \times D^{2}-7.42 \times D+4.75
$$

This condition is actually more strict than the TM condition given in equation (2).

In many applications it is important to achieve small modal birefringence, defined here as the difference between the effective indexes of the two orthogonally polarized modes, the horizontally polarized mode (quasi-TE) and the vertically polarized mode (quasi-TM), $\Delta N_{e f f}=N_{e f f}^{T E}-N_{e f f}^{T M}$. To achieve zerobirefringence in rib waveguides, an optimisation of waveguide dimensions is necessary [19]. As we are investigating a most common situation where there is an oxide upper cladding, the total birefringence is, however, the sum of the geometrical birefringence and stress induced birefringence [25]. Figure 2 shows the geometry of the modelled SOI rib waveguide. The buried oxide (BOX) layer is $1 \mu \mathrm{m}$ in thickness, while the waveguide height is $H=1.35 \mu \mathrm{m}$. These waveguide dimensions are chosen as we have previously reported optical filters based on such waveguides [e.g. 26,27]. The propagation loss of these waveguides were $<1$ $\mathrm{dB} / \mathrm{cm}$ [28], and we have recently shown that $0.7 \mu \mathrm{m}$ high rib waveguides can have the propagation loss as low as $\sim 0.2 \mathrm{~dB} / \mathrm{cm}$ [29]. The upper oxide cladding thickness in these simulations is in the range 0.1-3 $\mu \mathrm{m}$. The upper $\mathrm{SiO}_{2}$ layer produces a stress distribution within and near the $\mathrm{Si} \mathrm{rib}$, which in turn causes a change of the refractive index in both materials due to the photoelastic effect. We assume that the thickness of the upper cladding film on the rib sidewalls is $70 \%$ of that on the top, in order to simulate the film deposition used during the fabrication (figure 1). The stress induced birefringence can be expressed as [30]:

$$
\begin{aligned}
& \Delta n_{x}=n_{x}-n_{0}=-C_{1} \sigma_{x}-C_{2}\left(\sigma_{y}+\sigma_{z}\right) \\
& \Delta n_{y}=n_{y}-n_{0}=-C_{1} \sigma_{y}-C_{2}\left(\sigma_{x}+\sigma_{z}\right)
\end{aligned}
$$

where $\sigma_{x}, \sigma_{y}$, and $\sigma_{z}$ are principal components of the relative stress tensor, $n_{x}$ and $n_{y}$ are components of the material's refractive index, $n_{0}$ is the refractive index without stress, and $C_{l}$ and $C_{2}$ are the stress-optic constants related to the Young's modulus $(E)$, Poisson's ratio $(v)$, and the photoelastic tensor elements $\left(p_{11}\right.$ and $\left.p_{12}\right)$ as [30]:

$$
\begin{aligned}
& C_{1}=\frac{n^{3}}{2 E}\left(p_{11}-2 v p_{12}\right) \\
& C_{2}=\frac{n^{3}}{2 E}\left[-v p_{11}+(1-v) p_{12}\right]
\end{aligned}
$$

Table 1. Material parameters used in the calculations for the wavelength of $1550 \mathrm{~nm}$.

\begin{tabular}{cccccc}
\hline Material & $p_{11}$ & $p_{12}$ & Young's modulus $(\mathrm{GPa})$ & Poisson's ratio & $\begin{array}{c}\text { Thermal expansion } \\
\text { coefficient } \alpha\left(\mathrm{K}^{-1}\right)\end{array}$ \\
\hline $\mathrm{Si}$ & -0.101 & 0.0094 & 130 & 0.27 & $3.6 \times 10^{-6}$ \\
$\mathrm{SiO}_{2}$ & 0.16 & 0.27 & 76.7 & 0.186 & $5.4 \times 10^{-7}$ \\
\hline
\end{tabular}


The refractive index for the silicon guiding layer used in the model is 3.476 and the BOX and top cladding layer are 1.444, respectively, at an operating wavelength of $1550 \mathrm{~nm}$. The difference between the operating and reference temperature is assumed to be $-980 \mathrm{~K}$. Table 1 summarises other material parameters used in our simulations which are identical to those used in [25], where the influence of the top oxide cover was analysed for larger rib waveguides $(H=2.2 \mu \mathrm{m})$.

The stress distribution in the $\mathrm{Si}$ rib waveguide is calculated by the finite element method, which is implemented in a COMSOL programme [31]. Once the stress distribution is known, the local refractive index distribution can be evaluated using (5). The mesh used in our simulations was over 30000 elements providing good accuracy and reasonable calculation time. For the $\mathrm{SiO}_{2}$ upper and lower claddings, the inplane stress component in the uniform film far enough from the rib, is assumed to be $\sigma_{\text {film }}=-290 \mathrm{MPa}$. It should be noted that the primarily in-plane $(x)$ stress in the oxide film compresses the Si rib, resulting in a compressive stress in $x$-direction and higher tensile stress in the $y$-direction. This anisotropy is the main reason for the stress-induced birefringence. COMSOL is also used to solve Maxwell's equations for both quasi-TM and quasi-TE waveguide mode distributions and to determine the corresponding effective index values.

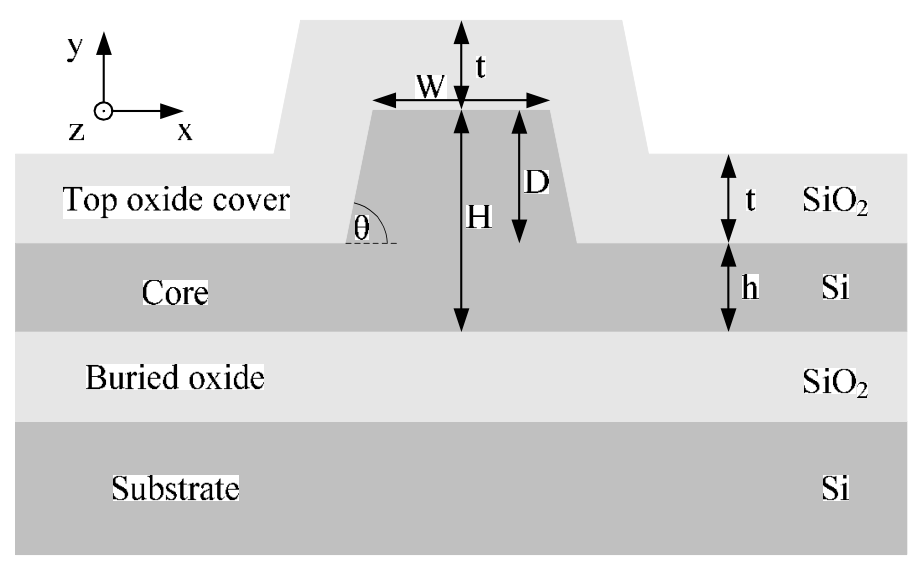

Figure 2. The cross section of an SOI rib waveguide ( $t$ is the top oxide layer thickness, $H$ is rib height, $D(D=H-h)$ is etch depth and $W$ is waveguide rib width

Figure 3 shows the influence of the oxide cover on the birefringence. For an air cover, the total birefringence is equal to the geometrical birefringence and it is positive for the majority of examples shown in figure 3 . Waveguides with relatively small rib widths can have negative geometrical birefringence. One such example ( $W=0.5 \mu \mathrm{m}, D=0.95 \mu \mathrm{m}$ ) is shown in figure 3. It can be seen that the oxide cover reduces the total birefringence and for certain waveguide geometries and oxide thicknesses it becomes negative. Therefore, by stress engineering, zero-birefringence(ZBR) can be achieved for a waveguide that otherwise has relatively large positive birefringence. It is worth noting that the position of birefringence curve depends strongly on the combination of $W$ and $D$. Hence, for some values of $W$ and $D, \Delta N=0$ cannot be achieved even for a thick oxide cover.

In order to investigate the polarization characteristics of the waveguide due to its geometry, the modal birefringence was evaluated whilst keeping the waveguide height and top oxide cover thickness constant during the simulation. The waveguide height is equal $1.35 \mu \mathrm{m}$, while the thickness of the top oxide cover is considered over a wide range of thickness from $0.1 \mu \mathrm{m}$ to $3.0 \mu \mathrm{m}$. The results are shown in figure 4 . It can be noticed that the increase of the top oxide cover thickness shifts the zero birefringence curves to smaller values of the etch depth. Also, the ZBR condition can be achieved for higher values of $W$, whilst the bottom part of the curve is 'saturated' (figure 4). For the upper part of the ZBR curve, that is for wider ribs, when increasing the oxide thickness the birefringence will become negative and therefore to make it zero again, the width of the rib needs to be increased in order to reduce the stress influence on the rib. On the other hand, for the bottom part of the ZBR curve, that is for narrower ribs, the mode resides mainly in the slab region and therefore the increase of the oxide thickness will not affect the birefringence significantly, hence the saturation in that region (figure 4). 


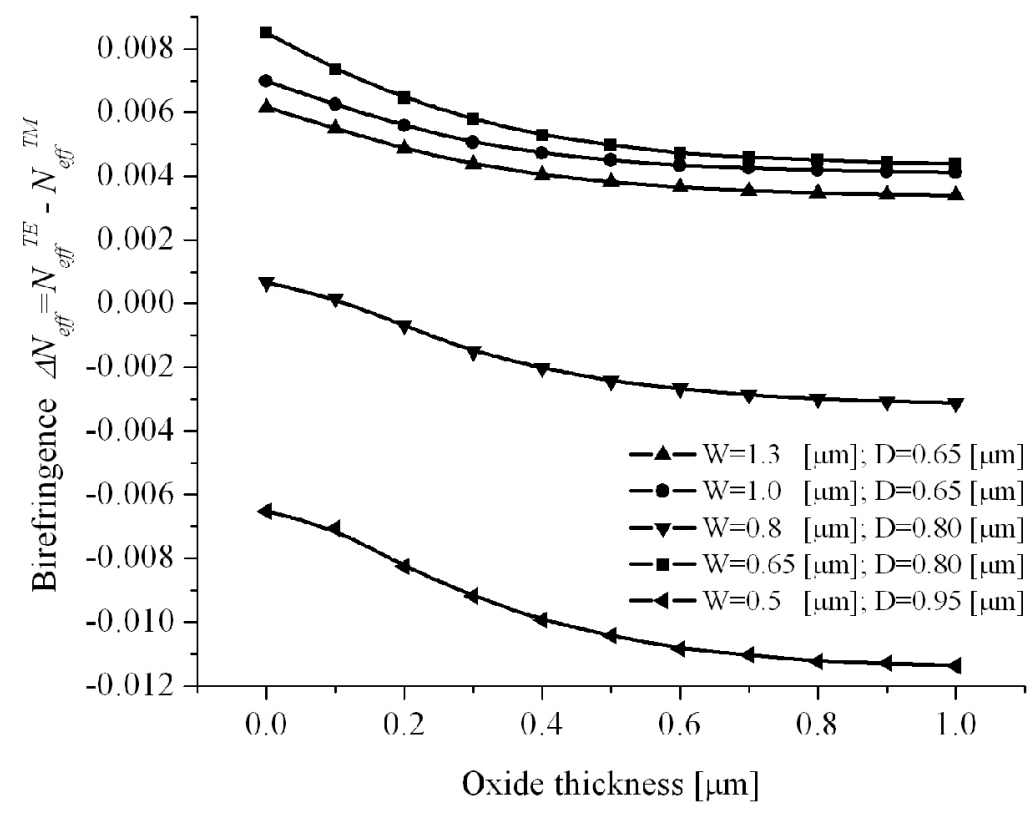

Figure 3. Waveguide birefringence as a function of top oxide cover thickness and waveguide dimensions $(\lambda=1.55 \mu \mathrm{m}, H=1.35 \mu \mathrm{m})$. Zero-birefringence condition is achieved for $W=D=0.8 \mu \mathrm{m}$ and for top oxide cover thickness of $\sim 120 \mathrm{~nm}$

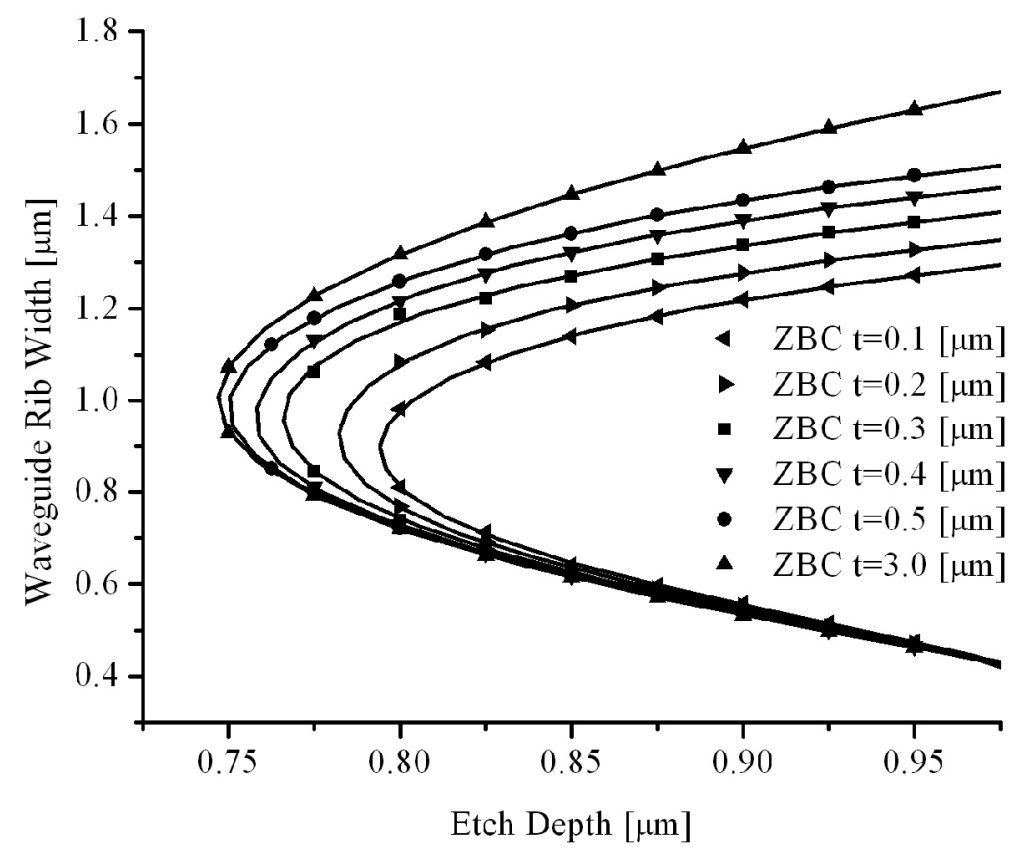

Figure 4. Zero birefringence condition $(\mathrm{ZBC})$ as a function of waveguide dimensions and top oxide cover thickness $(\lambda=1.55 \mu \mathrm{m}, H=1.35 \mu \mathrm{m})$

It is interesting to see what happens with the ZBR curve when the side wall angle is taken into account. Figure 5 shows two examples, one with vertical side walls $\left(\theta=90^{\circ}\right)$ and one with the angle of $\theta=80^{\circ}$ (figure 2 ), which is a typical variation in fabricated rib waveguides (figure 1). Figure 5 shows that the ZBR curve shifts to lower values of $W$ for angled sidewalls. When $\theta$ is decreased, for constant $W$ (width of the top of the rib), the width of the bottom of the rib will increase. In other words, the effective width of the rib will increase and therefore for constant oxide thickness, the ZBR condition will not be fulfilled. To obtain $\Delta N=0$, effective rib width needs to be decreased, which means that the ZBR condition will be fulfilled for narrower ribs. That is the reason why the ZBR curve shifts down in figure 5. In conclusion, in order to have both ZBR and SM conditions satisfied, $W$ and $D$ should be on the ZBR curve and equation (4) needs to be fulfilled. Therefore, careful design of waveguides needs to be carried out prior to fabrication, and good fabrication control is essential. 


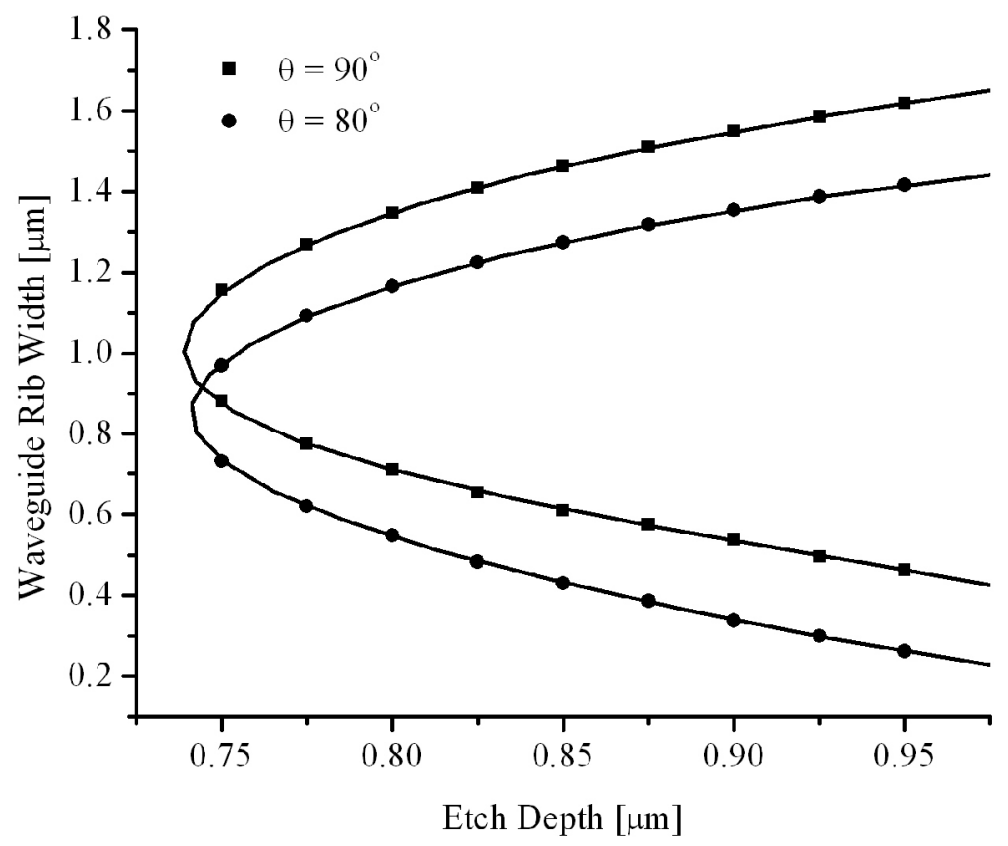

Figure 5. Influence of the side wall angle on the ZBR condition ( $\lambda=1.55 \mu \mathrm{m}, H=1.35 \mu \mathrm{m}, \underline{t=1 \mu \mathrm{m}})$

\section{Hollow-core waveguides}

Depending on the structure of the cladding, hollow core waveguides are classified in two categories: the Bragg-mirror cladding waveguides (e.g. [32]) and the ARROW (anti-resonant reflecting optical waveguide) waveguides (e.g. [16]). The Bragg-mirror cladding comprises alternating layers of two types of materials, the so called "bilayers" (figure 6a). In this case, light is totally reflected at the multilayer, for any incident angle or polarisation and the light can propagate through a low refractive index core. The multilayer cladding of the ARROW waveguides is designed to behave like a Fabry-Perot interferometric reflector. The number of layers in this case can vary from only 1 to $\mathrm{N}$.

The first step in the design of a hollow core waveguide (HCW) for MWIR is the choice of materials and the number of bilayers used for the Bragg-mirror cladding. To achieve the maximum reflectance at the interface of these two materials, it is desirable to have the greatest possible difference in their refractive indices at a specified wavelength. Also, in order to avoid any significant propagation loss, the absorption in both materials should obviously be as low as possible. Generally, a larger number of bilayers enables better confinement of light in the core, and provides smaller propagation losses. However, increasing the number of bilayers in the cladding increases the overall waveguide dimensions and further complicates the fabrication procedure. In order to choose an optimal number of bilayers, it is necessary to make a trade-off between tolerable propagation losses on the one hand and the fabrication complexity and waveguide dimensions on the other.

Materials that may be used in combination with silicon to form a Bragg-mirror cladding, must be technologically compatible with silicon. Usually materials considered for this purpose are silicon dioxide $\left(\mathrm{SiO}_{2}\right)$, silicon nitride $\left(\mathrm{SiN}_{\mathrm{x}}\right)$ and silicon-germanium alloys. Initial estimation of the HCW properties were carried out by running 2D BPM simulations. Simulations were carried out for various numbers of bilayers and for two specific wavelengths: $3.39 \mu \mathrm{m}$ and $10.6 \mu \mathrm{m}$. For a better comparison, the width of a hollow-core in all the simulations was equal to the wavelength (i.e. either $3.39 \mu \mathrm{m}$ or $10.6 \mu \mathrm{m}$ ). As expected, these simulations showed that the number of $\mathrm{SiGe} / \mathrm{Si}$ bilayers needed for low loss propagation at $3.39 \mu \mathrm{m}$ is much larger than the number of $\mathrm{Si} / \mathrm{SiO}_{2}$ bilayers, as the refractive index contrast in the $\mathrm{SiGe} / \mathrm{Si}$ cladding is not as high as in the $\mathrm{Si} / \mathrm{SiO}_{2}$. At $10.6 \mu \mathrm{m}$, however, the $\mathrm{Si} / \mathrm{SiO}_{2} \mathrm{HCW}$ system had high loss due to high absorption coefficient of $\mathrm{SiO}_{2}$ beyond $3.6 \mu \mathrm{m}$. Therefore, we focus on the analysis of $\mathrm{Si} / \mathrm{SiO}_{2} \mathrm{HCW}$ at the wavelength of 3-3.39 $\mu \mathrm{m}$, which is interesting for the gas detection applications [33].

The fabrication process for the proposed waveguide with $\mathrm{Si} / \mathrm{SiO}_{2}$ Bragg-mirror cladding is based on the Plasma Enhanced Chemical Vapour Deposition (PECVD) technique. After making a trench in a silicon substrate, alternating layers of $\mathrm{SiO}_{2}$ and $\mathrm{Si}$ are deposited, as depicted in figure $6 \mathrm{~b}$. Thus, a hollow waveguide 
core, with the sidewalls and the bottom cladding is made. A second wafer with a deposited upper cladding is then bonded to the first, eventually forming a hollow waveguide with the cross-section shown in figure 6a.

We have used a 3-D beam propagation method to calculate propagation losses in the $\mathrm{Si} / \mathrm{SiO}_{2}$-based $\mathrm{HCWs}$ for several different values of the core dimensions. Our aim was to further investigate the impact of the core dimensions on the propagation loss in the proposed waveguide structure. Specifically, we have performed 3D simulations for $\mathrm{Si} / \mathrm{SiO}_{2}$ based HCWs with the square-shaped hollow-core and 5 bilayers (figure 6). The propagation loss was calculated for the wavelength of $3 \mu \mathrm{m}$ and for three different waveguides with the core dimensions of $3 \mu \mathrm{m} \times 3 \mu \mathrm{m}, 5 \mu \mathrm{m} \times 5 \mu \mathrm{m}$ and $7 \mu \mathrm{m} \times 7 \mu \mathrm{m}$. The calculated propagation losses are presented in table 2.

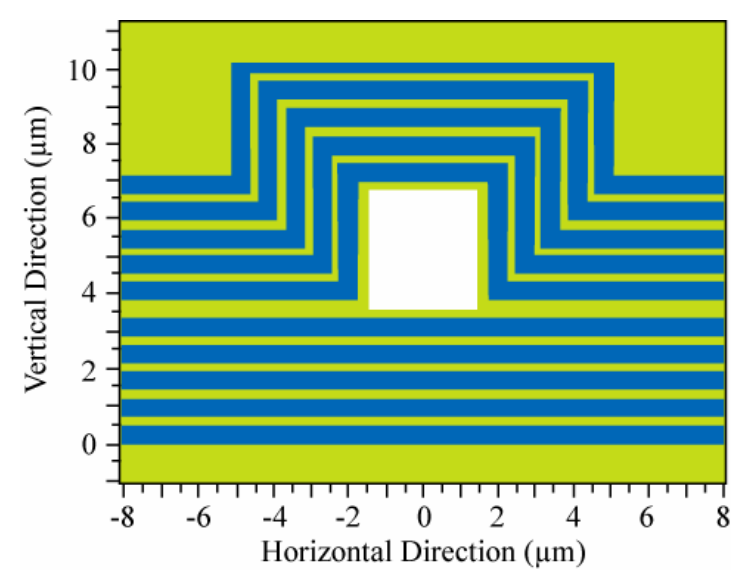

a)

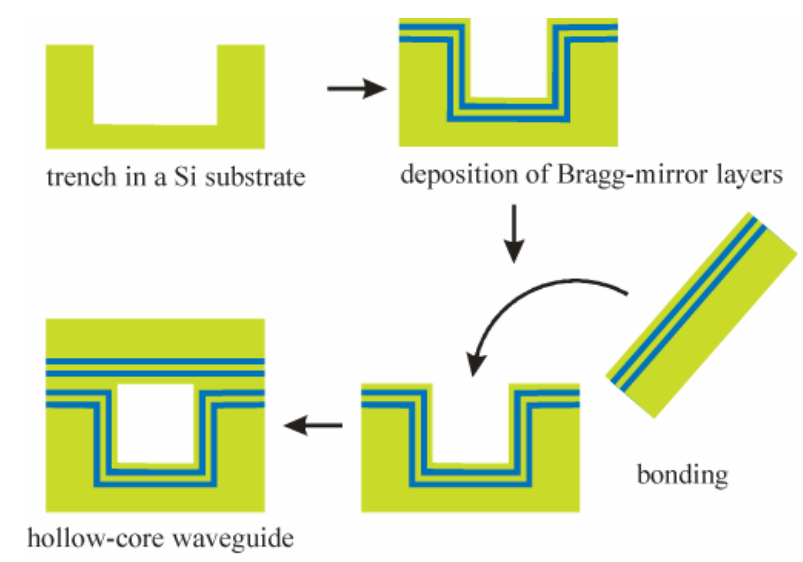

b)

Figure 6. a) Hollow core waveguide with Bragg cladding; b) fabrication of a hollow core waveguide

Table 2. Propagation losses for simulated waveguides with $5 \mathrm{Si} / \mathrm{SiO}_{2}$ bilayers, at $\lambda=3 \mu \mathrm{m}$.

\begin{tabular}{ll}
\hline $\begin{array}{l}\text { Core dimensions } \\
(\text { width } \times \text { height })\end{array}$ & $\begin{array}{l}\text { Propagation loss } \\
{[\mathrm{dB} / \mathrm{cm}]}\end{array}$ \\
\hline $3 \mu \mathrm{m} \times 3 \mu \mathrm{m}$ & 42.05 \\
$5 \mu \mathrm{m} \times 5 \mu \mathrm{m}$ & 4.72 \\
$7 \mu \mathrm{m} \times 7 \mu \mathrm{m}$ & 0.53 \\
\hline
\end{tabular}

These results indicate that the core dimensions have a significant impact on the propagation loss. Generally, as the core dimensions increase, the propagation loss decreases, which is consistent with the results reported for the ARROW waveguides [32]. Additional simulations were performed at the wavelength of $\lambda=3.39 \mu \mathrm{m}$ (table 3). For better comparison, core dimensions (width and height) were expressed as multiples of the wavelength.

Table 3. Propagation losses calculated for hollow-core waveguides with claddings based on $\mathrm{Si} / \mathrm{SiO}_{2}$ bilayers, at $\lambda=3.39 \mu \mathrm{m}$.

\begin{tabular}{lll}
\hline $\begin{array}{l}\text { Core dimensions } \\
\text { (width } \times \text { height) } \\
\text { [in wavelengths] }\end{array}$ & $\begin{array}{l}\text { Number of } \\
\text { bilayers in } \\
\text { cladding }\end{array}$ & $\begin{array}{l}\text { Propagation loss } \\
\text { [dB/cm] }\end{array}$ \\
\hline $2 \lambda \times 2 \lambda$ & 4 & 4.78 \\
$2 \lambda \times 2 \lambda$ & 5 & 2.41 \\
$3 \lambda \times 3 \lambda$ & 4 & 0.66 \\
$3 \lambda \times 3 \lambda$ & 5 & 0.17 \\
$4 \lambda \times 4 \lambda$ & 4 & 0.06 \\
\hline
\end{tabular}

These results show that waveguides with only 4 or 5 bilayers in the cladding, yet relatively large core dimensions of no less than three wavelengths in both width and height could exhibit propagation losses of 
less than $1 \mathrm{~dB} / \mathrm{cm}$. Also, it is obvious that the core dimensions have even greater impact on propagation losses than overall number of bilayers in the cladding.

These analyses were carried out for the ideal geometrical properties of the proposed square-shaped, hollowcore waveguides with a $\mathrm{Si} / \mathrm{SiO}_{2}$ Bragg-mirror cladding. Having in mind the fabrication procedure for such devices (as depicted in figure 6b), this specific geometrical shape of the waveguide is difficult to produce. It is reasonable to expect that there would be an air-filled gap at the interface between the two wafers. This gap would result in the waveguide having a cross section shown in figure 7. To investigate the impact of an air gap to the waveguide performance we have performed a set of simulations for the waveguide comprising 5 bilayers in the cladding and having the core dimensions of $3 \lambda \times 3 \lambda$, where $\lambda$ was once again $3.39 \mu \mathrm{m}$. We have calculated the propagation losses for three values of the air gap thickness and these results are presented in table 4.

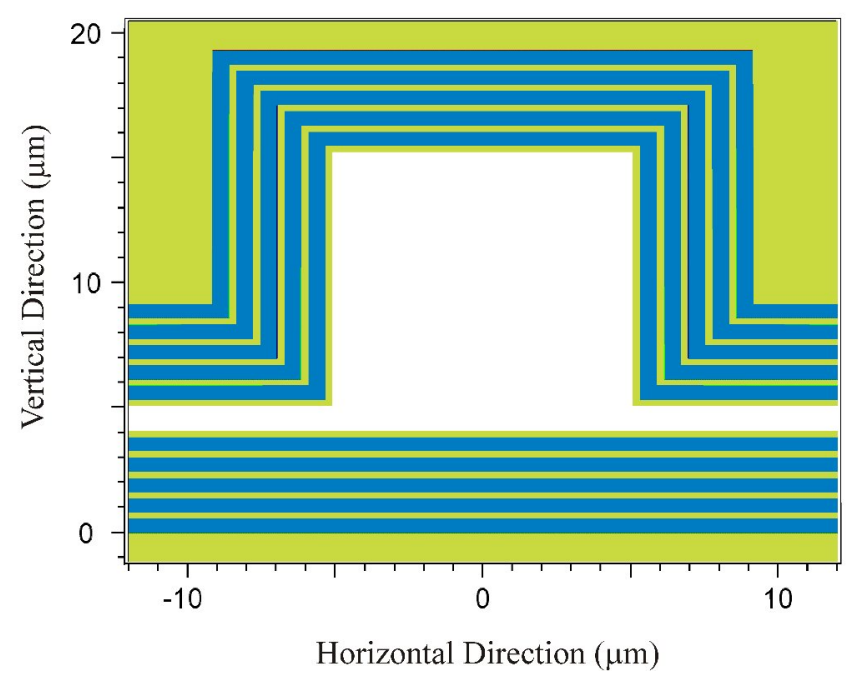

Figure 7. Hollow-core waveguide with a square cross-section and the air gap between the lower and the upper sections.

Table 4. Propagation losses for hollow-core waveguides with $5 \mathrm{Si} / \mathrm{SiO}_{2}$ bilayers in the cladding and an air gap between the lower and the upper segments $(\lambda=3.39 \mu \mathrm{m})$.

\begin{tabular}{lll}
$\begin{array}{l}\text { Core dimensions } \\
\text { (width } \times \text { height) } \\
\text { [in wavelengths] }\end{array}$ & air gap & $\begin{array}{l}\text { Propagation } \\
\text { loss }[\mathrm{dB} / \mathrm{cm}]\end{array}$ \\
\hline $3 \lambda \times 3 \lambda$ & $1.0 \mu \mathrm{m}$ & 0.44 \\
$3 \lambda \times 3 \lambda$ & $1.5 \mu \mathrm{m}$ & 0.99 \\
$3 \lambda \times 3 \lambda$ & $2.0 \mu \mathrm{m}$ & 1.83 \\
\hline
\end{tabular}

As expected, these results show that as the air gap widens, the propagation loss increases. However, even for a $1.5 \mu \mathrm{m}$ thick gap, the propagation loss is still lower than $1 \mathrm{~dB} / \mathrm{cm}$. These results, however, need to be confirmed by using a more accurate simulation method such as 3D FDTD.

\section{Free standing waveguides}

Silicon waveguide with an air cladding will be a viable structure for terahertz applications, as silicon is low loss in the 24-100 $\mu \mathrm{m}$ wavelength range. We have fabricated such waveguides using Proton Beam Writing (PBW). PBW is a new direct-writing process that uses a focused beam of $\mathrm{MeV}$ or sub-MeV protons to pattern nano/micro structures in a wide range of materials. The fabrication process of PBW is similar to ebeam writing, nevertheless it offers some interesting and unique advantages. Due to the large mass mismatch between protons and electrons $\left(\mathrm{m}_{\mathrm{p}} / \mathrm{m}_{\mathrm{e}}=1800\right)$, protons penetrate deeper into the semiconductor while maintaining a straight path. Unlike the high energy secondary electrons generated during e-beam writing, the secondary electrons induced by the primary protons have low energies, and therefore results in a limited range, which minimizes the proximity effects. This opens the way to allow the PBW to be a direct-writing 
lithography technique for fabrication of high aspect ratio structures. Another unique characteristic of proton beams is that their penetration depth is well defined and can be controlled by changing the proton energy. Finally, the irradiated dose is tunable in a pre-defined pattern, by pausing the proton beam for different times at selected locations. In this way, any pattern of localized damage can be built up. This feature is used to produce three-dimensional multilevel structures in bulk p-type silicon [34].

$$
\text { High Medium Low }
$$

(a)

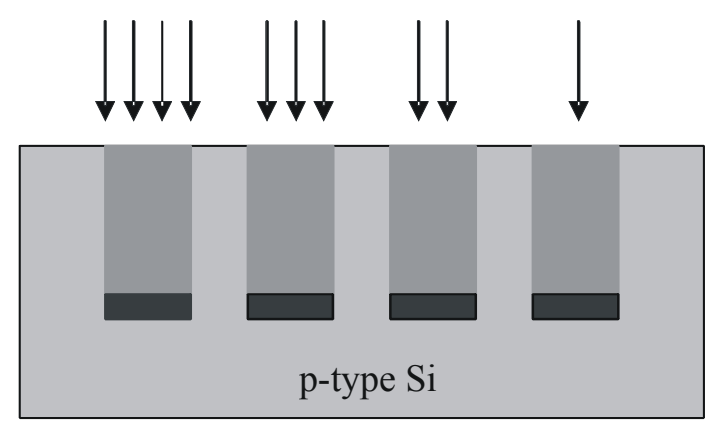

(b)

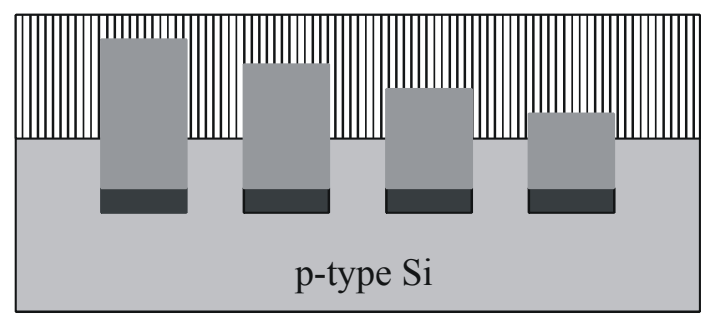

(c)

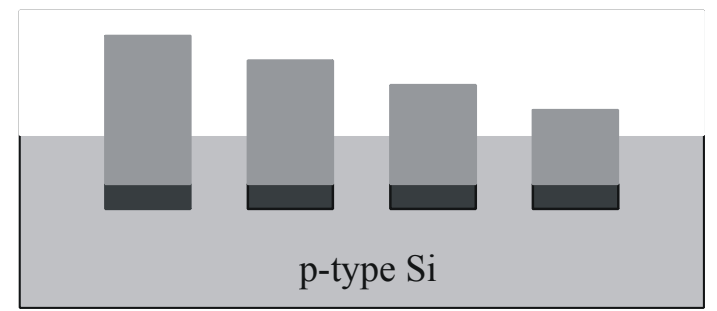

Figure 8. Schematic diagram of the experimental procedure. (a) $2 \mathrm{MeV}$ protons irradiation into p-type Si followed by (b) porous silicon formation in hydrofluoric (c) porous silicon removal in diluted $\mathrm{KOH}$ solution reveals the final structure

Typically, a well-focused proton beam of $200 \mathrm{~nm}$ resolution is used to irradiate the silicon sample (figure $8 \mathrm{a}$ ). The scanning pattern is controlled by a computer program. For feature areas which are less than $500 \times 500 \mu \mathrm{m}^{2}$, the magnetic scan is used directly. When the structure is bigger than $500 \mu \mathrm{m}$, the movement of the sample is provided by a piezo-stage over lengths of up to $2.5 \mathrm{~cm}$. As protons propagate through silicon, they undergo a series of collisions with electrons. Protons are deflected away from an ideal straight path, resulting in a beam cross section increasing in size with depth. The energy deposited due to nuclear collisions per unit length is fairly constant during the first half of the penetration depth but increases sharply at the end of the range, because the speed of protons is much lower than their initial value, thereby increasing the probability of nuclear collisions with target atoms. By tuning the scanning time, different fluences are delivered. The irradiated sample is then anodized in a dilute solution of hydrofluoric acid, by passing an electrical hole current through it with an applied bias, resulting in porous silicon formation at the surface (figure $8 \mathrm{~b}$ ). The damaged regions inhibit this process; the result is a significant reduction in the rate of producing porous silicon in the proton irradiated regions as compared to the unirradiated region. The final structure produced after etching is a three dimensional representation of the scanned area. This area determines the dimensions of the final structure. The etching time and current controls the height of the structure (figure 8c).

If two proton energies are selected, a lower proton energy scanned bridge can be fabricated with higher proton energy irradiated pillars. One example is shown in figure $9 \mathrm{a}$ where $2 \mathrm{MeV}$ implantation is used for the 
pillar formation. After prolonged etching beyond the end of the range (figure 9b), since the lower energy has a shorter range, the isotropic etching process starts to undercut at a shallower etch depth while the pillars with higher energy irradiation continue to increase in height (figure 9c). In this way, freestanding microstructures can be fabricated in a single etch step. This would have required multiple processing steps if a conventional lithography technique is used. Figure 10 shows a sideview of a $0.5 \mathrm{MeV}$ bridge and a $2 \mathrm{MeV}$ pillar demonstrating the undercutting of the waveguide.

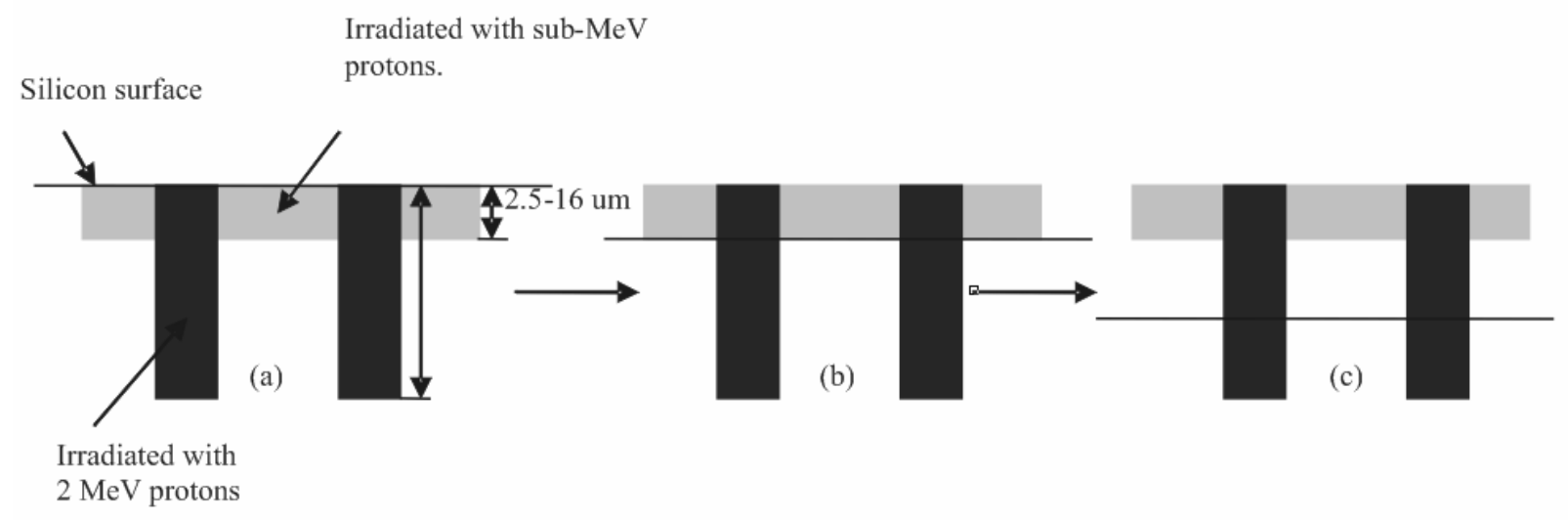

Figure 9. Fabrication process of freestanding structure (a) Bridge irradiated with sub-MeV protons, and pillars usually irradiated with $2 \mathrm{MeV}$ protons. (b) Etching reaches the end of range of the sub-MeV protons.

(c) The isotropic etching process undercut the region irradiated with sub-MeV protons

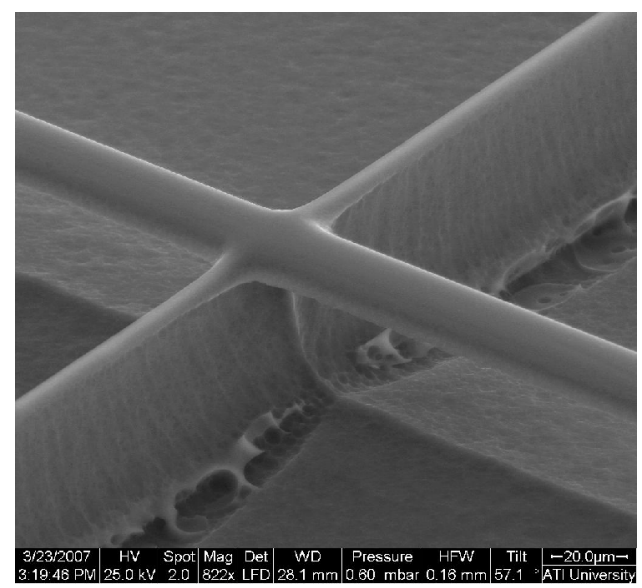

Figure 10. Sideview of a freestanding bridge with supporting pillar

Recently, we have demonstrated freestanding waveguides in Si. An SEM image of the first freestanding test chip is shown in figure 11a [35]. The waveguide was irradiated by $1 \mathrm{MeV}$ protons and it had a "tear-drop" cross sectional shape with a height of $17 \mu \mathrm{m}$, and width of $3.7 \mu \mathrm{m}$ at the top and $8 \mu \mathrm{m}$ at the bottom, because of the beam spreading at the end of the range.

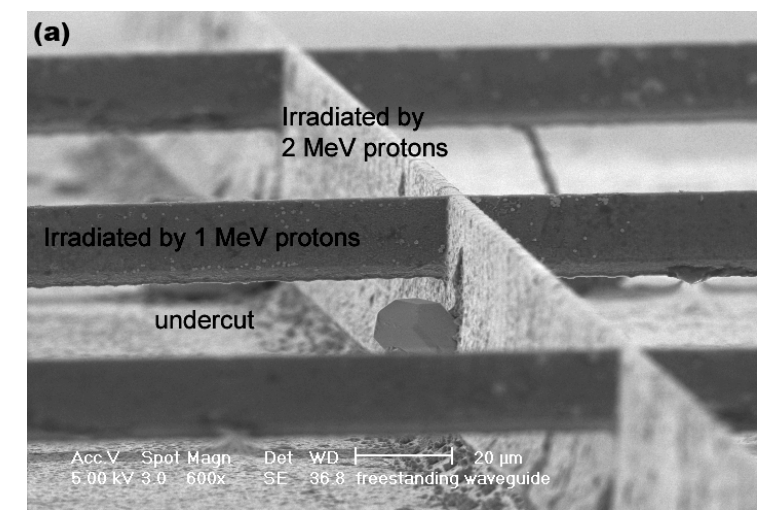

(a)

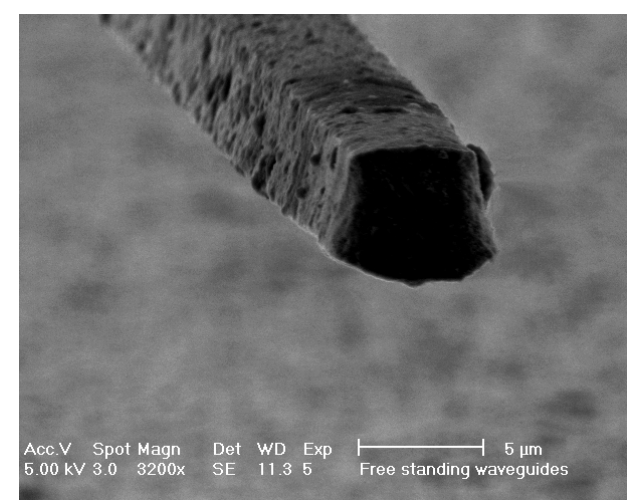

(b)

Figure 11. a) free standing waveguide from the first test chip [37]; b) new free standing waveguide with square cross section 
The subsequent batch of freestanding waveguide structures with improved cross-section have been fabricated by employing $0.5 \mathrm{MeV}$ proton energy. The proton fluence delivered to the sample has been $5 \times 10^{14}$ protons $/ \mathrm{cm}^{2}$. After irradiation, the sample has been electrochemically etched in a 1:1 electrolyte mixture of $48 \% \mathrm{HF}$ and ethanol at a current density of $40 \mathrm{~mA} / \mathrm{cm}^{2}$. Once the silicon becomes porous, it can be removed by a dilute potassium hydroxide solution, producing a waveguide with an approximately square profile in the cross section. A scanning electron microscope (SEM) image of the waveguide cross section is shown in figure $11 \mathrm{~b}$. The waveguide has a height of $5.5 \mu \mathrm{m}$ and width of $5 \mu \mathrm{m}$ at the top and $6 \mu \mathrm{m}$ at the bottom. Relatively high propagation loss of $\sim 10 \mathrm{~dB} / \mathrm{cm}$ is mainly due to protons irradiation damage, sidewall roughness induced during the electrochemical etching process, and intersection loss between the waveguide and the pillars. Annealing the sample in a high temperature and wet oxygen environment, oxidizes the porous silicon film and forms silicon dioxide. The oxidation process anneals lattice damage, and smoothes the sidewall at the same time [36]. The oxide can be left as a cladding or subsequently removed in buffered HF [37]. In this way, the propagation loss of freestanding waveguide can be reduced. By annealing the waveguides at the temperature of $\sim 500^{\circ} \mathrm{C}$ we expect that more than $90 \%$ of the defects caused by the proton irradiation of silicon will be anneal out [38] and that the propagation loss could be in the 4-6 dB/cm range, similar to the work presented in [39]. Further decrease of the propagation loss can be expected by smoothing the side walls by using different etching conditions and scanning parameters, as well as post-fabrication treatment such as oxidation. This and the reduction of intersection loss between the waveguide and pillars will be the main focus of our future work in this area.

\section{Conclusions}

In this paper we have presented waveguides for three different wavelength ranges. The design rules for rib SOI waveguides at the wavelength of $1.55 \mu \mathrm{m}$ have been given. Both influences of the oxide thickness and side wall angles have been taken into account. It has been shown that by stress engineering, zerobirefringence can be achieved for a waveguide that has otherwise relatively large birefringence. As single mode and zero-birefringence propagation significantly depend on the waveguide's dimensions and geometry, and the top oxide cover, a careful design prior to the fabrication needs to be carried out.

The 3-5 $\mu \mathrm{m}$ wavelength range is interesting for several reasons, one being the sensing of different gases as a number of gases have absorption peaks in this range. A hollow-core waveguide with an $\mathrm{Si} / \mathrm{SiO}_{2}$ Bragg-mirror cladding, optimised for this spectral range has been proposed and simulated. Superior characteristics of the $\mathrm{Si} / \mathrm{SiO}_{2}$-based cladding have been demonstrated in comparison with the claddings based on $\mathrm{Si} / \mathrm{SiGe}$. The significance of the exact waveguide geometry modelling and its hollow-core size has been stressed. The core size has proven to be an essential parameter and it is suggested that its both horizontal and vertical dimensions should be at least three times the wavelength for which the waveguide is designed. In addition, it has been demonstrated that for a sufficiently large core dimensions, the waveguides comprising only 4 bilayers in the cladding could achieve propagation losses less than $1 \mathrm{~dB} / \mathrm{cm}$. Also, the impact of an air gap between the waveguide sections has been analyzed. It has been shown that the propagation losses increase as the gap widens, yet these losses could still be less than $1 \mathrm{~dB} / \mathrm{cm}$ if the gap is less than $1.5 \mu \mathrm{m}$ thick.

In our future work, we plan to carry-out simulations that would include a more realistic waveguide core geometry with the slanted side walls (which is unavoidable in real devices due to the nature of the fabrication process), i.e. with a trapezoidal-shaped core cross section. Also, the impact of surface roughness and layer thickness variations needs to be included in theses analyses to provide more complete theoretical predictions. Single mode conditions will also be analysed.

Finally, for far-infrared wavelength region, where silicon has still low propagation loss, a free standing waveguide has been proposed. We have improved the waveguides cross-section compared to the first batch of devices by reducing the implant energy to $0.5 \mathrm{MeV}$. Annealing and the reduction of intersection loss between the waveguide and pillars by fabrication of narrower pillars will be the main focus of our future work in order to reduce the propagation loss of these waveguides.

It can be seen that the silicon waveguide technology could be used for a very wide range of wavelengths by applying different waveguide geometries and fabrication techniques. The core dimensions scale up with the wavelength and the propagation losses are still relatively large for less mature fabrication techniques. 


\section{Acknowledgements}

The authors are grateful to Intel Corporation for the fabrication of rib waveguides, and to Jasna Crnjanski from the University of Belgrade for the simulation of hollow core waveguides.

\section{References}

[1] Soref R A and Lorenzo J P 1986 IEEE J. Quantum Electron. QE-22 873

[2] Soref R A and J. P. Lorenzo 1985 Electron. Lett. 21953

[3] Albares D J and Soref R A 1987 Proc. SPIE 70424

[4] Soref R A, Namavar F and Lorenzo J P 1990 Opt. Lett. 15270

[5] Cortesi E, Namavar F and Soref R A 1989 IEEE SOS/SOI Technology Conference (Cat. No.89CH2796-

1), 3-5 Oct. 1989. Stateline, NV, USA: IEEE, 109

[6] Kurdi B N and Hall D G 1988 Opt. Lett. 13175

[7] Rickman A, Reed G T, Weiss B L and Namavar F 1992 IEEE Photon. Technol. Lett. 4633

[8] Schmidtchen J, Splett A, Schuppert B and Petermann K 1991 IEEE International SOI Conference

Proceedings (Cat. No.91CH3053-6) 142

[9] Chan S P, Png C E, Lim S T, Passaro V M N and Reed G T 2005 J. Lightwave Technol. 231573

[10] Yamada K, Fukuda H, Watanabe T, Tsuchizawa T, Shoji T, and Itabashi S-I 2005 Proc. LEOS $2^{\text {nd }}$

Group IV Photonics, Antwerp, Belgium, 186

[11] Vlasov Y A and McNab S J 2004 Opt. Exress 121622

[12] Dumon P et al. 2004 IEEE Photon. Technol. Lett. 161328

[13] Bogaerts W et al. 2005 J. Lightwave Technol. 23401

[14] Bernini R, Campopiano S and Zeni L 2002 IEEE J. Sel. Top. Quantum Electron 8106

[15] Yin D, Schmidt H, Barber J and Hawkins A 2004 Opt. Express 122710

[16] Schmidt H, Yin D, Barber J P and Hawkins A R 2005 IEEE J. Sel. Top. Quantum Electron. 11519

[17] Lou S S, Wang M S and Chen C C 2004 Opt. Express 126589

[18] Soref R A, Emelett S J and Buchwald W R 2006 J. Opt. A: Pure Appl. Opt. 8840

[19] Raghunathan V, Borlaug D, Rice R R and Jalali B 2007 Proc. LEOS $4^{\text {th }}$ Group IV Photonics, Tokyo, Japan, PD2

[20] Soref R A, Schmidtchen J and Petermann K 1991 IEEE J. Quantum Electron. 271971

[21] Pogossian S P, Vescan L and Vonsovici A 1998 J. Lightwave Technol. 161851

[22] Powell O 2002 J. Lightwave Technol. 20, 1851

[23] Vivien L, Laval S, Dumont B, Lardenois S, Koster A and Cassan E 2002 Opt. Commun. 21043

[24] Lousteau J, Furniss D, Seddon A, Benson T M, Vukovic A and Sewell P 2004 J. Lightwave Technol. 22 1923

[25] Ye W N, Xu D-X, Janz S, Cheben P, Picard M-J, Lamontagne B and Tarr N G 2005 J. Lightwave Technol. 231308

[26] Headley W R, Reed G T, Paniccia M, Liu A, Cohen O and Hak D 2004 Appl. Phys. Lett. 855523

[27] Timotijevic B D, Gardes F Y, Headley W R, Reed G T, Paniccia M J, Kohen O, Hak D and Masanovic G Z 2006 J. Opt. A: Pure Appl. Opt. 8 S473

[28] Headley W 2005 PhD thesis, University of Surrey

[29] Gardes F Y et al. 2008 Proc. SPIE 6898-23

[30] Huang M 2003 Int. J. Sol. Structures 401615

[31] www.comsol.com

[32] Mashanovich G, Pucker G, Kompocholis C, Lui A, Crnjanski J, Stankovic S, Passaro V M N, Matavulj

P and Reed G T 2006 Proc 14th Telecom. Forum TELFOR 2006 (Belgrade, Serbia) 357

[33] Mulrooney J, Clifford J, Fitzpatrick C and Lewis E 2007 Sensors and Actuators A 136104

[34] Teo E J, Breese M B H, Tavernier E P, Bettiol A A, Watt F, Liu M H and Blackwood D J 2004 Appl.

Phys. Let 843202

[35] Yang P Y, Mashanovich G Z, Gomez-Morilla I, Headley W R, Reed G T, Teo E J, Blackwood D J, Breese M B H and Bettiol A A 2007 Appl. Phys. Lett. 90241109

[36] Lee K L, Lim D R, Luan H-C, Agarwal A, Foresi J and Kimerling L C, 2000 Appl. Phys. Lett., 771617

[37] Shieh S-Y and Evans J W 1994 J.Vac.Sci.Technol.B 121422

[38] Day A C, Horne W E and Arimura 1980 IEEE Trans. Nucl. Sci. 271665

[39] Teo E J et al. 2008 Opt. Express 16573 